\section{Ingeniería}

CONTENIDO

Artículos

Análisis numérico de un sistema de aire acondicionado empleando
mallas estructuradas y no estructurades A

Funciones de regresión para caudales extremos en la Vertiente Rojas, Nazareth; Aguilar, José Francisco; Solís, Hernán. Evaluación de daños por agresión ambiental en viviendas
de concreto reforzado. Solís, Rómel; Moreno, Eric; Jiménez, Felipe; Rosas, Victorino.

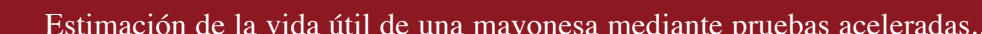
Estimación de la vida útil de una mayonesa
García, Claudia; Molina, Manuel.

Inestabilidad oscilatoria de tensión debido a los motores de inducción …….....................65-75 rde, Gustavo; Araya, Eddie.

Estabilidad y colapso de tensión en sistemas eléctricos Araya, Eddie.

Obtención de pulpa a la soda antraquinona de Tectona grandis creciendo en Costa Rica...........................

Protocolo evaluador para el manejo de desechos y la seguridad ocupacional
aplicado al laboratorio clínico del Hospital San Francisco de Asís de Grecia. Ruiz, Francisco.

9. Euler: su contex
Vargas, Celso.

10. Reserva de resistencia de edificios porticados de concreto armado disenaados conforme al ACI-318/ IBC 2006 ........ Análisis y comentarios

Herrera, Rodolfo.

breve semblanza.

Trabajos de Graduación 2007

Lista de proyectos de graduación de grado y posgrado

Normas

$\sum_{\text {EDTORAL }}$

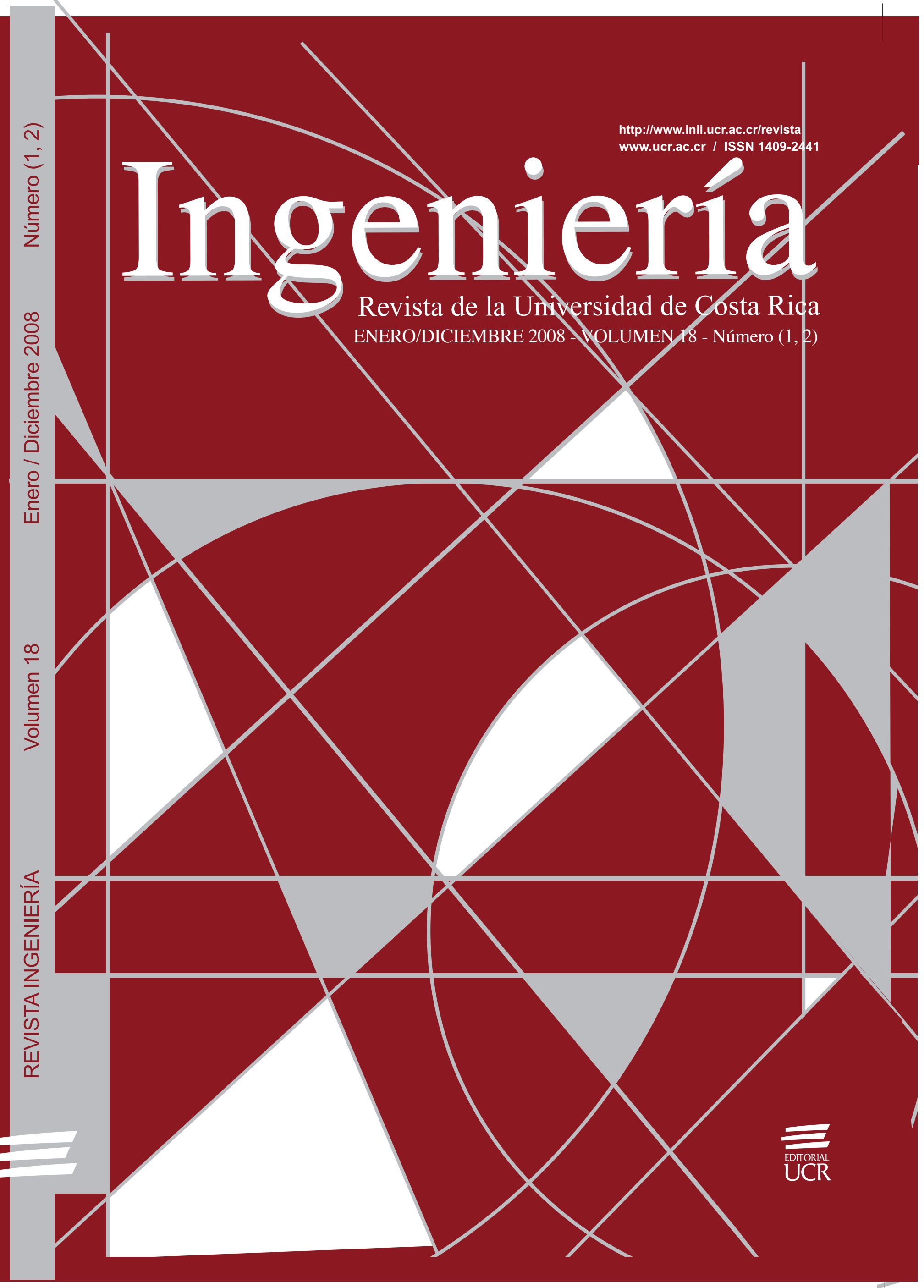




\title{
FUNCIONES DE REGRESIÓN PARA CAUDALES EXTREMOS EN LA VERTIENTE PACÍFICA DE COSTA RICA
}

\author{
Nazareth Rojas Morales \\ José Francisco Aguilar Pereira \\ Hernán Solís Bolaños
}

\begin{abstract}
Resumen
Las variaciones en la cantidad y la calidad de los recursos hídricos están relacionadas con el clima, este determina qué cantidad de agua, en exceso o en déficit, recibirá una región. En Costa Rica este recurso es abundante, pero con distribuciones muy variadas durante las dos estaciones climáticas. Este artículo aborda el estudio en eventos máximos por medio de la hidrología estadística. La referencia base es la establecida en el boletín 17B de la Interagency Advisory Committee on Water Data (IACWD, 1982) donde se definen las guías para determinar la frecuencia de caudales de inundación, el cual es un procedimiento estándar en los Estados Unidos. La obtención de las ecuaciones de regresión para diferentes probabilidades de excedencia aplicables a la vertiente Pacífica, se obtuvieron mediante la conjugación de la distribución de probabilidad Log-Person III y de regresión lineal y exponencial múltiple. Los parámetros que se relacionaron para la obtención de las ecuaciones son el caudal máximo anual, el área de drenaje y la precipitación promedio anual de las subcuencas establecidas, tomando como punto de cierre las estaciones limnigráficas. Los registros de caudal fueron aportados por el Instituto Costarricense de Electricidad (ICE) y los de precipitación por el Instituto Metereológico Nacional (IMN) y el ICE. El análisis estadístico realizado mediante las series de datos de caudal máximo anual de las estaciones en estudio, mostró buenos resultados. En consecuencia, se establece que existe una relación real entre las variables, debido a la confiabilidad de las muestras de datos y de los resultados obtenidos. Las ecuaciones generadas pueden ser utilizadas como un método empírico para el cálculo de caudal para los diferentes períodos de retorno analizados en la vertiente del Pacífico.
\end{abstract}

Palabras clave: hidrología, análisis de frecuencia, estimación de caudales, análisis estadístico, análisis de regresión, periodo de retorno, funciones de regresión.

\begin{abstract}
Changes in the quantity and quality of water resources are related to the climate, which determines the quantity of water, in excess or in deficit, which will receive a region. In Costa Rica this resource is abundant, but water distributions change dramatically during the two climatic seasons. The present article studies maximum events by means of statistical hydrology. The technical background is established in the bulletin 17B of the Interagency Advisory Committee on Water Data (IACWD, 1982), which guides the determination of flood flow frequency that constitutes a standard procedure in the United States. The regression equations were obtained for different probabilities that are applicable to the Costa Rican Pacific coast by means of the combination of the distribution of probability Log-Person III and of multiple linear and exponential regressions. The parameters that were related for the equations are the maximum annual flow, the area of drainage and the annual average precipitation of the established sub basins defined by limnigraphic stations locations. The flow records were provided by the ICE and the records of precipitation by the IMN and the ICE. The statistical analysis was performed by means of the series of information of maximum annual flow of the stations in study showed good results. As a consequence it is established that a real relation exists between the variables, due to the reliability of the samples of information and the obtained results. The generated equations can be used as an empirical method for the calculation of flow for the different analyzed return periods in the pacific coast.
\end{abstract}

Keywords: hydrology, frequency analysis, discharge estimation, statistic analysis, regression analysis, return period. 


\section{INTRODUCCIÓN}

Estimar de manera precisa el daño potencial de una inundación es un elemento clave en los programas nacionales de emergencias. Los tomadores de decisión de alta jerarquía requieren, a la hora de tomar acciones, de información técnica especializada y confiable. En este sentido, parte de este artículo aborda un procedimiento que busca determinar la frecuencia de flujos de inundación el cual es base, entre otros, para la modelación hidráulica requerida para la consecución de las planicies de inundación. Es relevante indicar la importancia de la calidad de la información que requiere el especialista, la cual es fundamental para lograr que este insumo tenga características consistentes y precisas.

En diciembre de 1967, el Departamento de Recursos Hídricos de los Estados Unidos publica el boletín No. 15, el cual es considerado como el pionero en esta temática y se tituló "Una técnica uniforme para determinar frecuencias de flujos de inundación”. Esta técnica fue acogida, a nivel federal, por el estado y por los gobiernos locales para la planificación de los recursos hídricos y la administración de tierras. A partir de esta fecha se han realizado actualizaciones hasta el boletín 17B en el año 1982, desarrollado por el Subcomité de Hidrología de la USGS (United States Geological Survey), donde amplía los métodos de análisis y promueve que su aplicación sea uniforme, precisa y consistente (IACWD, 1982).

Es importante resaltar que hay elementos de riesgo e incertidumbre que son inherentes en cualquier análisis de frecuencia de inundaciones, y se hace más relevante en países en desarrollo, por lo que el análisis estadístico por sí solo, no resolverá los problemas para su determinación. Por tanto, el juicio del especialista experimentado debe ser un componente fundamental en el análisis hidrológico.

La metodología para la obtención de ecuaciones de regresión para el cálculo de caudal máximo anual con diferentes períodos de retorno, aplicada en este artículo, ha sido aprobada por la Agencia Federal de Manejo de Emergencias de los Estados Unidos, por sus siglas en inglés, FEMA. Por tanto el objetivo planteado en el artículo es determinar las ecuaciones de regresión para el cálculo de caudal máximo anual con diferentes períodos de retorno, para ser aplicadas en cualquier cuenca que se ubique en la vertiente Pacífica de Costa Rica.

\section{MATERIALES Y MÉTODOS}

\subsection{Definición del área de análisis}

La selección de la región de trabajo fue determinada considerando la información de caudal y de precipitación. En este sentido, el ICE puso a disposición la información de tres importantes cuencas de la vertiente del Pacífico: río Tempisque, río Grande de Tárcoles y río Grande de Térraba.

Se seleccionó la vertiente Pacífica para realizar el estudio de investigación, ya que la información es suficientemente representativa de las condiciones climáticas de dicha Vertiente: condiciones relativamente secas en el Pacífico Norte, condiciones medias en el Pacífico Central y condiciones húmedas en el Pacífico Sur. El Cuadro 1 muestra la información respectiva para cada una de las estaciones.

\subsection{Análisis estadísticos de la información limnigráfica}

El análisis de la confiabilidad de los datos se realizó por valores salidos extremos y límites de confianza, utilizando la metodología de la Oficina de Coordinación de Datos de Agua del Departamento de Interior de los Estados Unidos presentados por el Subcomité de Hidrología en las Guías para determinar la frecuencia de flujo en inundaciones.

\subsubsection{Ajuste de parámetros estadísticos si se detectan valores extremos}

Se detectaron valores salidos extremos en las estaciones y se realizaron las correcciones respectivas en los valores de la media, desviación estándar y coeficiente de sesgo. En las ecuaciones 
Cuadro 1. Estaciones limnigráficas.

\begin{tabular}{lcccccc}
\multicolumn{1}{c}{ Cuenca } & Estación & Número & Área $\left.\mathbf{( k m}^{2}\right)$ & Latitud & Longitud & Río \\
Río Tempisque & Guardia & 741901 & 955,00 & 282,800 & 362,100 & Tempisque \\
Río Tempisque & Coyolar & 741906 & 128,20 & 294,400 & 374,050 & Tempisque \\
Río Grande Térraba & Palmar & 983101 & 4766,7 & 105,090 & 595,857 & Térraba \\
Río Grande Térraba & Caracaucho & 983107 & 1133,3 & 110,160 & 624,565 & Coto Brus \\
Río Grande Térraba & Brujo & 983103 & 2401,2 & 119,995 & 616,066 & Térraba \\
Río Grande Térraba & La Cuesta & 983109 & 835,50 & 137,000 & 580,140 & General \\
Río Grande Térraba & Rivas & 983108 & 317,90 & 157,680 & 576,123 & Chirripó \\
Río Grande Tárcoles & Nuestro Amo & 842424 & 734,00 & 212,834 & 506,488 & Tárcoles \\
Río Grande Tárcoles & Tacares & 842402 & 201,50 & 220,637 & 502,375 & Tárcoles \\
Río Grande Tárcoles & Balsa & 842406 & 1638,0 & 212,281 & 495,308 & Tárcoles \\
Río Grande Tárcoles & Garita & 842422 & 649,40 & 218,087 & 498,538 & Tárcoles
\end{tabular}

Fuente: (ICE, 2004)

(1) y (2) se presentan las funciones del umbral inferior y del umbral superior de los valores salidos extremos.

$X_{H}=\bar{X}+K_{N} S$

$X_{L}=\bar{X}-K_{N} S$

donde:

$X_{H}$ Umbral de los valores altos salidos extremos en unidades logarítmicas

$X_{L}$

$\bar{X}$ Promedio de los logaritmos de los valores de caudal.

$S$ Desviación estándar de la muestra de los logaritmos de los valores de caudal.

$K_{N} \quad$ Valores de $K$ que depende del número de valores de la muestra $N$.

\subsubsection{Ajuste para los datos altos salidos}

Una vez que se obtiene dentro de una muestra un dato salido extremo, se procede a eliminar de la misma dicho valor, de manera que se tiene que realizar de nuevo cada uno de los procedimientos indicados hasta este momento. Una vez realizados los análisis, se procede a aplicar los siguientes procesos.

Se obtiene el valor del Factor de Peso (W), mediante la ecuación (3).

$W=\frac{H-Z}{N+L}$

donde:

$H$ es el período histórico, el cual es la cantidad de años de registro, por ejemplo, si se tiene un período de registro del año 1892 a 1973, el valor de $\mathrm{H}$ es igual a 82 , pues se incluyen cada uno de los límites del período.

$L$ corresponde al valor de datos bajos salidos extremos.

$N \quad$ número de datos excluyendo el número de datos salidos extremos.

Z número de datos altos salidos extremos.

2.2.3 Los estadísticos de los valores salidos extremos

Los estadísticos de los valores salidos extremos se ajustan por medio de las siguientes ecuaciones (4), (5) y (6). 
Ajuste del promedio de los datos de la $Q=10^{\log Q}$
muestra

$\bar{M}=\frac{W N M+\sum X_{Z}}{H-W L}$

donde:

$M$ corresponde a $X$ de la muestra

$\sum X_{2}$ es el valor de la sumatoria de los logaritmos de los datos altos salidos extremos.

Ajuste de la desviación estándar de la muestra

Este ajuste se realiza mediante la ecuación (5).

$\bar{S}^{2}=\frac{W(N-1) S^{2}+W N(M-\bar{M})^{2}+\sum\left(X_{Z}-\bar{M}\right)^{2}}{H-W L-1}$

\section{Cálculo del Coeficiente de Sesg}

Finalmente, se corrigieron los registros que presentaron valores salidos extremos y sus correspondientes valores estadísticos (ver ecuación (6) )..

2.3 Obtención de los caudales estimados con base en los parámetros estadísticos ajustados

Aplicando la ecuación de Chow y el Método de Log-Pearson III (IACWD, 1982), se obtuvieron los caudales para cada estación limnigráfica para los períodos de retorno (T) de (100, 50, 20, 10, 2 y 1) años.

$$
\log Q=\bar{X}+K S
$$

donde:

$Q \quad$ Caudal (m3/s)

K Coeficiente de Chow

X Media

$S \quad$ Desviación estándar

\subsection{Desarrollo de las funciones de regresión}

\subsubsection{Selección de variables independientes}

Se seleccionaron como variables independientes el área de la cuenca y la precipitación media para determinar la variable dependiente del caudal por ser las que frecuentemente conducen a valores mayores del coeficiente de correlación y por su sentido físico dominante en el fenómeno del escurrimiento superficial.

2.4.2 Determinación del área de drenaje empleando herramientas de Sistemas de Información Geográfica (SIG).

Se utilizaron herramientas de análisis espacial y de tres dimensiones teniendo como insumo curvas de nivel cada $20 \mathrm{~m}$ para las cuencas de los ríos en mención, a fin de crear los modelos de elevación digital que son la base para los procesos de análisis. Por otro lado, se usaron datos de información espacial tanto para las estaciones limnigráficas como para las pluviométricas.

$$
\bar{G}=\frac{H-W L}{(H-W L-1)(H-W L-2) \bar{S}^{3}}\left[\frac{W(N-1)(N-2) S^{3} G}{N}+3 W(N-1)(M-\bar{M}) S^{2}+W N(M-\bar{M})^{3}+\sum\left(X_{Z}-\bar{M}\right)^{3}\right]
$$




\section{RESULTADOS}

\subsection{Modelo de Elevación Digital (MED)}

El modelo de elevación digital es de tipo red irregular triangular (por sus siglas en inglés, TIN) la cual se generó a partir de las curvas de nivel y el límite de la cuenca. Este modelo de superficie establece como unidad base geométrica un polígono triangular, cuyos vértices tienen atributos espaciales lo cual permitió que la información de las cuencas de los ríos Tárcoles, Tempisque y Grande de Térraba muestren sus rasgos lineales del relieve y del drenaje, permitiendo modelar la superficie del terreno geomorfológicamente y será la referencia para los estudios de las subcuencas mostradas en el Cuadro 1. Los modelos del TIN generados se pueden apreciar en las Figuras 1, 2 y 3 , donde los Cuadros 2, 3 y 4 definen los valores de las características de las cuencas estudiadas.

\subsection{Análisis de datos salidos extremos altos y bajos de las cuencas}

Se encontró que las estaciones Nuestro Amo y Tacares de la cuenca del río Grande de Tárcoles, mostraron valores salidos extremos altos. Por otra parte, la Estación Guardia, de la cuenca del río Tempisque y la Estación Garita, de la cuenca del río Grande de Tárcoles mostraron valores salidos extremos bajos. En consecuencia, se procedió a realizar los ajustes correspondientes. Se muestran los resultados detallados para la Estación Guardia, y un resumen de los resultados de las otras estaciones.

3.2.1 Análisis de la Estación Guardia de la cuenca del río Tempisque.

Los registros de caudal máximo anual cubren un período de 43 años.

\section{Prueba de datos salidos extremos.}

Al comparar los valores de caudal alto y caudal bajo con la lista de registro, se observa que existe un valor bajo salido, este valor corresponde a $30,7 \mathrm{~m} \mathrm{3/s} \mathrm{(Cuadro} \mathrm{5).}$

En vista de que existe un valor bajo salido, se procede a realizar el ajuste de este dato, obteniéndose los resultados presentados en el Cuadro 6.

Cuadro 2. Valores de las características de la cuenca del río Grande de Tárcoles.

$\begin{array}{lc}\quad \text { Característica } & \text { Valor } \\ \text { Área } & 2135 \mathrm{~km}^{2} \\ \text { Perímetro } & 234 \mathrm{~km} \\ \text { Índice de compacidad } & 1,44 \\ \text { Elevación máxima } & 2900 \mathrm{~m} . \mathrm{s} . \mathrm{n} . \mathrm{m} . \\ \text { Elevación mínima } & 0 \text { m.s.n.m } \\ \text { Elevación media } & 1063,53 \mathrm{~m} . \mathrm{s} . \mathrm{n} . \mathrm{m} . \\ \text { Longitud de cauce } & 111,7 \mathrm{~km} \\ \text { Pendiente media del río } & 2,15 \%\end{array}$

Fuente: (ICE, 2004)

Cuadro 3. Valores de las características de la cuenca del río Grande de Térraba.

\begin{tabular}{lc}
\multicolumn{1}{r}{ Característica } & Valor \\
Área & $5076,8 \mathrm{~km}^{2}$ \\
Perímetro & $380 \mathrm{~km}$ \\
Índice de compacidad & 1,49 \\
Elevación máxima & $3820 \mathrm{~m} . \mathrm{s} . \mathrm{n} . \mathrm{m}$. \\
Elevación mínima & $5 \mathrm{~m} . \mathrm{s} . \mathrm{n} . \mathrm{m}$. \\
Elevación media & $997,5 \mathrm{~m} . \mathrm{s.n} . \mathrm{m}$. \\
Longitud del cauce & $160 \mathrm{~km}$ \\
Pendiente media del río & $2,2 \%$
\end{tabular}

Fuente: (ICE, 2004)

Cuadro 4. Valores de las características de la cuenca del río Tempisque.

$\begin{array}{lc}\quad \text { Característica } & \text { Valor } \\ \text { Área } & 3407,8 \mathrm{~km}^{2} \\ \text { Índice de compacidad } & 1,51 \\ \text { Perímetro } & 315 \mathrm{~km} \\ \text { Elevación máxima } & 1860 \mathrm{~m} . \mathrm{s} . \mathrm{n} . \mathrm{m} \\ \text { Elevación media } & 930 \mathrm{~m} . \mathrm{s} . \mathrm{n} . \mathrm{m} \\ \text { Elevación mínima } & 0 \mathrm{~m} . \mathrm{s} . \mathrm{n} . \mathrm{m} . \\ \text { Longitud del cauce } & 138 \mathrm{~km} \\ \text { Pendiente media del río } & 1,4 \%\end{array}$

Fuente: (ICE, 2004) 


\section{Cuenca Río Grande de Tárcoles}

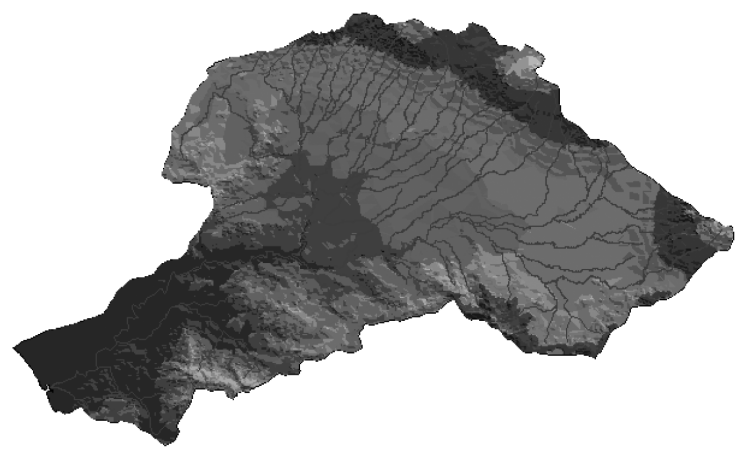

Distribución de elevaciones

Rango de elevaciones

Rango de elevaciones

$2589-2900$
$2278-2589$

$1967-2278$

$1656-1967$

$1344-1656$

$1344-1656$
$1033-1344$

$722-1033$

$411-722$

$100-411$

Figura 1. Distribución de elevaciones, cuenca río Grande de Tárcoles. Fuente: (La autora y los autores).

\section{Cuenca Río Grande de Térraba}

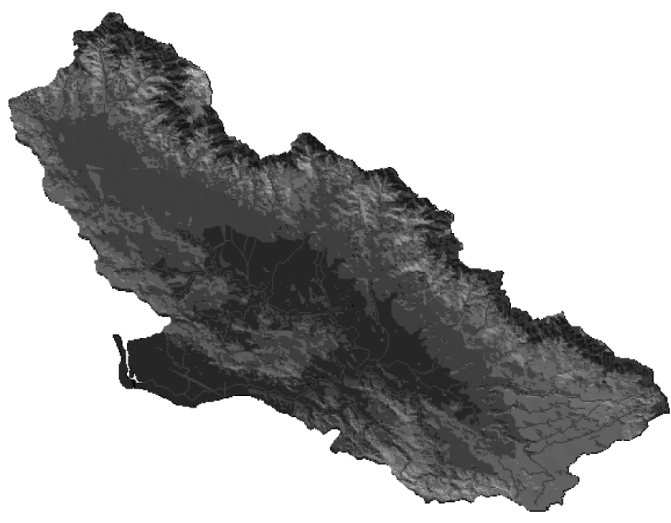

$$
\triangle \text { Ríos }
$$

Distribución de las elevaciones Rango de elevaciones $3300-3700$ $2900-3300$ $2500-2900$

$2100-2500$

$1700-2100$

$1300-1700$

$900-1300$

$500-900$

$100-500$

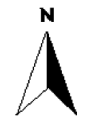

Figura 2. Distribución de elevaciones, cuenca río Grande de Térraba. Fuente: (La autora y los autores). 


\section{Cuenca Río Tempisque}

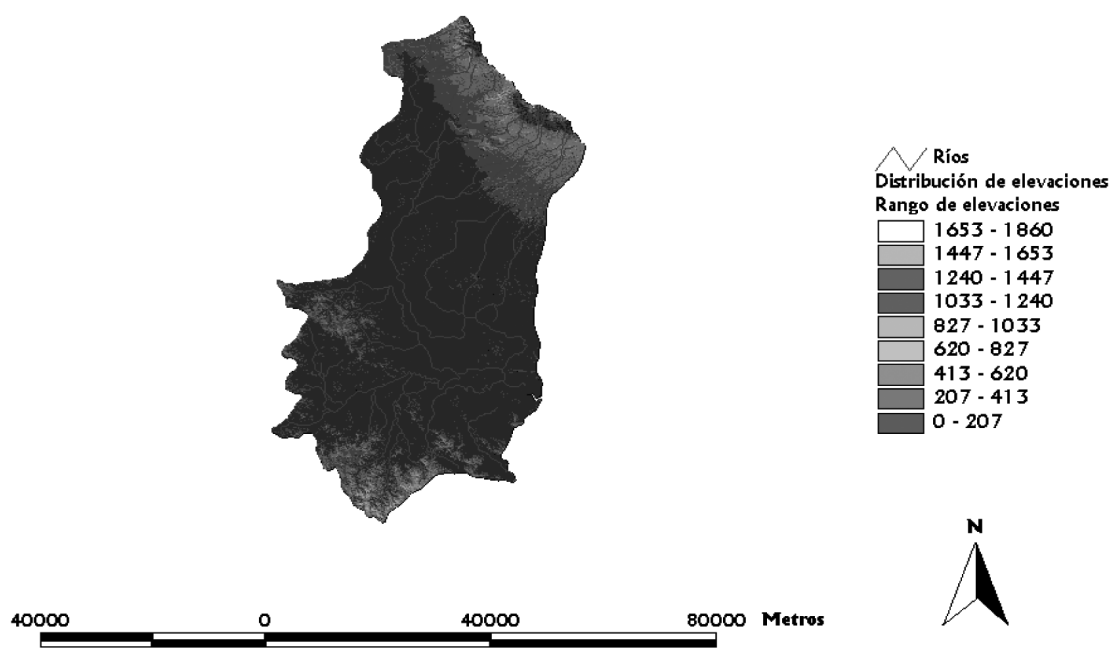

Figura 3. Distribución de elevaciones, cuenca del río Tempisque.

Fuente: (La autora y los autores).

Cálculo y ajuste de la Curva de Frecuencia

El cálculo de la Curva de Frecuencia se basa en la estadística del Cuadro 6 (Cuadro 7).

Los registros de caudal máximo anual cubren un período de veintidós años.

\section{Prueba de datos salidos extremos}

Al realizar el análisis respectivo se determinó que existe un dato alto salido el cual corresponde al registro No. 18 igual a $1614 \mathrm{~m} 3 / \mathrm{s}$ (Cuadro 8).

Al eliminar este valor del registro total se vuelven a realizar los cálculos respectivos, de donde se obtienen los resultados que se presentan en el Cuadro 9.

\subsubsection{Análisis de la Estación Tacares de la cuenca del río Grande de Tárcoles.}

Los registros de caudal máximo anual cubren un período de 49 años.
Prueba de datos salidos extremos.

Al realizar el análisis respectivo se determinó que existe un dato alto salido el cual corresponde al registro No. 3, igual a $271 \mathrm{~m} 3 / \mathrm{s}$ (Cuadro 10).

Después de eliminar el dato correspondiente se procede a realizar el respectivo análisis y los resultados se muestran en el Cuadro 11

\subsubsection{Análisis de la Estación Garita de la cuenca del río Grande de Tárcoles.}

Los registros de caudal máximo anual cubren un período de 23 años, no hubo registros para los años 1985 y 1986.

\section{Prueba de datos salidos extremos}

Al realizar el análisis respectivo se determinó que el registro 20 correspondiente a $29,6 \mathrm{~m} 3 / \mathrm{s}$ representa un dato bajo salido y los resultados se muestran en el Cuadro 12. 
Cuadro 5. Resultados.

\begin{tabular}{lccc}
\multicolumn{1}{c}{ Variable } & Símbolo & Unidad & Resultado \\
Promedio & $\mathrm{M}$ & & 2,6441 \\
Desviación estándar & $\mathrm{S}$ & & 0,4063 \\
Coeficiente de sesgo & $\mathrm{G}$ & & $-0,76$ \\
Coeficiente & $\mathrm{KN}$ & & 2,71 \\
Logaritmo de caudal alto & $\mathrm{Log} \mathrm{QH}$ & & 3,7451 \\
Caudal salido alto & $\mathrm{QH}$ & $\left(\mathrm{m}^{3} / \mathrm{s}\right)$ & 5560 \\
Logaritmo de caudal bajo & $\mathrm{Log} \mathrm{QL}$ & & 1,5431 \\
Caudal salido bajo & $\mathrm{QL}$ & $\left(\mathrm{m}^{3} / \mathrm{s}\right)$ & 35,00
\end{tabular}

Fuente: (La autora y los autores).

Cuadro 6. Resultados

\begin{tabular}{lccc}
\multicolumn{1}{c}{ Variable } & Símbolo & Unidad & Resultado \\
Promedio & $\mathrm{M}$ & & 2,6716 \\
Desviación estándar & $\mathrm{S}$ & & 0,3683 \\
Coeficiente de sesgo & $\mathrm{G}$ & $-0,48$ \\
Coeficiente & $\mathrm{KN}$ & & 2,70 \\
Logaritmo de caudal alto & $\mathrm{Log} \mathrm{QH}$ & & 3,6661 \\
Caudal salido alto & $\mathrm{QH}$ & $\left(\mathrm{m}^{3} / \mathrm{s}\right)$ & 4635 \\
Logaritmo de caudal bajo & $\mathrm{Log} \mathrm{QL}$ & & 1,6772 \\
Caudal salido bajo & $\mathrm{QL}$ & $\left(\mathrm{m}^{3} / \mathrm{s}\right)$ & 48,00
\end{tabular}

Fuente: (La autora y los autores)

Cuadro 7. Valores de la curva de frecuencia, análisis de la Estación Nuestro Amo de la cuenca del río Grande de Tárcoles.

$\begin{array}{ccccccc}\text { Probabilidad } & \text { Período } & \mathbf{M} & \mathbf{S} & \text { KGw } & \text { Log Q } & \begin{array}{c}\mathbf{Q} \\ \left(\mathbf{m}^{\mathbf{3} / \mathbf{s})}\right.\end{array} \\ & \mathbf{T} & & & & & 49 \\ 0,99 & 1,01 & 2,6716 & 0,3683 & -2,6717 & 1,6876 & 153 \\ 0,90 & 1,11 & 2,6716 & 0,3683 & -1,3218 & 2,1848 & 502 \\ 0,50 & 2,00 & 2,6716 & 0,3683 & 0,0797 & 2,7010 & 1320 \\ 0,10 & 10,00 & 2,6716 & 0,3683 & 1,2192 & 3,1206 & 1672 \\ 0,05 & 20,00 & 2,6716 & 0,3683 & 1,4975 & 3,2231 & 2139 \\ 0,02 & 50,00 & 2,6716 & 0,3683 & 1,7885 & 3,3303 & 2495 \\ 0,01 & 100,00 & 2,6716 & 0,3683 & 1,9696 & 3,3970 & \end{array}$

Fuente: (La autora y los autores). 
Cuadro 8. Resultados.

\begin{tabular}{lccc}
\multicolumn{1}{c}{ Variable } & Símbolo & Unidad & Resultado \\
Promedio & $\mathrm{M}$ & & 2,7698 \\
Desviación estándar & $\mathrm{S}$ & & 0,1760 \\
Coeficiente de sesgo & $\mathrm{G}$ & & 0,69 \\
Coeficiente & $\mathrm{KN}$ & & 2,43 \\
Logaritmo de caudal alto & $\mathrm{Log} \mathrm{QH}$ & & 3,1973 \\
Caudal salido alto & $\mathrm{QH}$ & $\left(\mathrm{m}^{3} / \mathrm{s}\right)$ & 1575 \\
Logaritmo de caudal bajo & $\mathrm{Log} \mathrm{QL}$ & & 2,3423 \\
Caudal salido bajo & $\mathrm{QL}$ & $\left(\mathrm{m}^{3} / \mathrm{s}\right)$ & 220
\end{tabular}

Fuente: (La autora y los autores).

Cuadro 9. Resultados

$\quad$ Variable
Promedio
Desviación estándar
Coeficiente de sesgo
Coeficiente
Logaritmo de caudal alto
Caudal salido alto
Logaritmo de caudal bajo
Caudal salido bajo

$\begin{array}{ccc}\text { Símbolo } & \text { Unidad } & \text { Resultado } \\ \mathrm{M} & & 2,7489 \\ \mathrm{~S} & & 0,1499 \\ \mathrm{G} & & 0,26 \\ \mathrm{KN} & 2,41 \\ \mathrm{Log} \mathrm{QH} & & 3,1098 \\ \mathrm{QH} & \left(\mathrm{m}^{3} / \mathrm{s}\right) & 1288 \\ \mathrm{Log} \mathrm{QL} & & 2,3880 \\ \text { QL } & \left(\mathrm{m}^{3} / \mathrm{s}\right) & 244\end{array}$

Fuente: (La autora y los autores).

Al eliminar el dato bajo salido se realiza de nuevo el análisis, obteniéndose los resultados mostrados en el Cuadro 13.

El modelo de regresión lineal y el exponencial múltiple, relacionan el caudal máximo anual, la precipitación media anual y el área de drenaje, en el cual estos dos últimos valores se obtienen de los resultados de los modelos de elevación digital respectivos.

A continuación se presentan los resultados tanto de área de drenaje como de precipitación promedio para cada una de las subcuencas establecidas a partir de las estaciones limnigráficas en estudio, además se indica cuál es el área de drenaje establecida por el ICE, para cada una de las subcuencas.
3.3 Datos de precipitación media y área para las subcuencas

\subsubsection{Cuenca del río Tempisque}

\section{Subcuenca de la Estación Guardia}

Área de drenaje $\left(\mathrm{km}^{2}\right)$ (Modelo de elevación digital ): 979,56

Área de drenaje $\left(\mathrm{km}^{2}\right)$ (ICE): 955,00

Precipitación promedio anual (mm): 1 747,38

\section{Subcuenca de la Estación Coyolar}

Área de drenaje $\left(\mathrm{km}^{2}\right)$ (Modelo de elevación digital ): 95,49

Área de drenaje $\left(\mathrm{km}^{2}\right)$ (ICE): 128,20 
Cuadro 10. Resultados.

\begin{tabular}{lccc}
\multicolumn{1}{c}{ Variable } & Símbolo & Unidad & Resultado \\
Promedio & $\mathrm{M}$ & & 1,8780 \\
Desviación estándar & $\mathrm{S}$ & & 0,1515 \\
Coeficiente de sesgo & $\mathrm{G}$ & & 0,66 \\
Coeficiente & $\mathrm{KN}$ & & 2,75 \\
Logaritmo de caudal alto & $\mathrm{Log} \mathrm{QH}$ & & 2,2952 \\
Caudal salido alto & $\mathrm{QH}$ & $\left(\mathrm{m}^{3} / \mathrm{s}\right)$ & 197 \\
Logaritmo de caudal bajo & $\mathrm{Log} \mathrm{QL}$ & & 1,4608 \\
Caudal salido bajo & $\mathrm{QL}$ & $\left(\mathrm{m}^{3} / \mathrm{s}\right)$ & 29,0
\end{tabular}

Fuente: (La autora y los autores)

Cuadro 11. Resultados

\begin{tabular}{lccc}
\multicolumn{1}{c}{ Variable } & Símbolo & Unidad & Resultado \\
Promedio & $\mathrm{M}$ & & 1,8780 \\
Desviación estándar & $\mathrm{S}$ & & 0,1515 \\
Coeficiente de sesgo & $\mathrm{G}$ & & 0,66 \\
Coeficiente & $\mathrm{KN}$ & & 2,75 \\
Logaritmo de caudal alto & $\mathrm{Log} \mathrm{QH}$ & & 2,2952 \\
Caudal salido alto & $\mathrm{QH}$ & $\left(\mathrm{m}^{3} / \mathrm{s}\right)$ & 197 \\
Logaritmo de caudal bajo & $\mathrm{Log}$ QL & & 1,4608 \\
Caudal salido bajo & $\mathrm{QL}$ & $\left(\mathrm{m}^{3} / \mathrm{s}\right)$ & 29,0
\end{tabular}

Fuente: (La autora y los autores).

Precipitación promedio anual (mm): 1 795,08.

\subsubsection{Cuenca del río Grande de Térraba}

\section{Subcuenca de la Estación Palmar}

Área de drenaje $\left(\mathrm{km}^{2}\right)$ (Modelo de elevación digital ): 4 533,75

Área de drenaje (km2) (ICE): 4 766,70

Precipitación promedio anual (mm): 3 028,10

\section{Subcuenca de la Estación Caracaucho}

Debido a problemas con la altimetría, -es decir como en la zona donde se ubica la estación es muy plana, no se generaron las suficientes curvas de nivel como para que el modelo estableciera toda la cuenca su totalidad- por ende, el análisis se realizó dividiendo a la misma en tres áreas adyacentes, luego se estableció el promedio de la precipitación de las tres.
Área de drenaje $\left(\mathrm{km}^{2}\right)$ (Modelo de elevación digital ): 1 553,31

Área de drenaje $\left(\mathrm{km}^{2}\right)$ (ICE): 1 133,30

Precipitación promedio anual (mm): 2 885,17

\section{Subcuenca de la Estación EI Brujo}

Área de drenaje $\left(\mathrm{km}^{2}\right)$ (Modelo de elevación digital ): 2 397,15

Área de drenaje $\left(\mathrm{km}^{2}\right)$ (ICE): 2 401,20

Precipitación promedio anual (mm): 3 118,07

\section{Subcuenca de la Estación La Cuesta}

Área de drenaje $\left(\mathrm{km}^{2}\right)$ (Modelo de elevación digital ): 831,42

Área de drenaje $\left(\mathrm{km}^{2}\right)$ (ICE ): 835,50

Precipitación promedio anual (mm ): 3 156,98

\section{Subcuenca de la Estación Rivas}

Área de drenaje $\left(\mathrm{km}^{2}\right)$ (Modelo de elevación digital ): 184,50 
Cuadro 12. Resultados

$\quad$ Variable
Promedio
Desviación estándar
Coeficiente de sesgo
Coeficiente
Logaritmo de caudal alto
Caudal salido alto
Logaritmo de caudal bajo
Caudal salido bajo

Símbolo Unidad

\section{Resultado}

M

2,4991

S $\quad 0,2737$

G $\quad-2,60$

$\mathrm{KN} \quad 2,45$

Log QH

3,1692

$\mathrm{QH} \quad\left(\mathrm{m}^{3} / \mathrm{s}\right) \quad 1476$

Log QL $\quad 1,8291$

QL $\quad\left(\mathrm{m}^{3} / \mathrm{s}\right) \quad 67,0$

Fuente: (La autora y los autores).

Cuadro 13. Resultados

$\quad$ Variable
Promedio
Desviación estándar
Coeficiente de sesgo
Coeficiente
Logaritmo de caudal alto
Caudal salido alto
Logaritmo de caudal bajo
Caudal salido bajo

$\begin{array}{ccc}\text { Símbolo } & \text { Unidad } & \text { Resultado } \\ \text { M } & & 2,5458 \\ \mathrm{~S} & & 0,1609 \\ \mathrm{G} & & -0,74 \\ \mathrm{KN} & 2,43 \\ \mathrm{Log} \text { QH } & & 2,9367 \\ \mathrm{QH} & \left(\mathrm{m}^{3} / \mathrm{s}\right) & 864 \\ \mathrm{Log} \mathrm{QL} & & 2,1550 \\ \mathrm{QL} & \left(\mathrm{m}^{3} / \mathrm{s}\right) & 143\end{array}$

Fuente: (La autora y los autores)

Cuadro 14. Valores de caudal, área y precipitación para el período de retorno de cien años.

\begin{tabular}{|c|c|c|c|c|}
\hline Estación & Número & $\begin{array}{c}\text { Caudal }(\mathbf{Q}) \\
\left(\mathrm{m}^{3} / \mathbf{s}\right)\end{array}$ & $\begin{array}{c}\text { Área (A) } \\
\left(\mathbf{k m}^{2}\right)\end{array}$ & $\begin{array}{c}\text { Precipitación media }(\mathbf{P}) \\
(\mathbf{m m} / \mathbf{a n ̃ o})\end{array}$ \\
\hline Guardia & 741901 & 2487 & 955 & 1747,38 \\
\hline Coyolar & 741906 & 1142 & 128,2 & 1795,08 \\
\hline Palmar & 983101 & 11305 & 4766,7 & 3028,10 \\
\hline Caracaucho & 983107 & 3323 & 1133,3 & 2885,17 \\
\hline Brujo & 983103 & 5629 & 2401,2 & 3118,07 \\
\hline La Cuesta & 983109 & 2509 & 835,5 & 3156,98 \\
\hline Rivas & 983108 & 579 & 317,9 & 3450,78 \\
\hline Amo & 842424 & 1832 & 734 & 2082,74 \\
\hline Tacares & 842402 & 253 & 201,5 & 2632,52 \\
\hline Balsa & 842406 & 1539 & 1638 & 2206,17 \\
\hline Garita & 842412 & 678 & 637,2 & 2400,32 \\
\hline
\end{tabular}

Fuente: (La autora y los autores) 
Área de drenaje $\left(\mathrm{km}^{2}\right)$ (ICE): 317,90

Precipitación promedio anual (mm): 3 450,78

\subsubsection{Cuenca del río Grande de Tárcoles}

\section{Subcuenca de la Estación Nuestro Amo}

Área de drenaje $\left(\mathrm{km}^{2}\right)$ (Modelo de elevación digital ): 804,96

Área de drenaje $\left(\mathrm{km}^{2}\right)$ (ICE): 734,00

Precipitación promedio anual (mm): 2 082,74

\section{Subcuenca de la Estación Tacares}

Área de drenaje $\left(\mathrm{km}^{2}\right)$ (Modelo de elevación digital ): 231,39

Área de drenaje $\left(\mathrm{km}^{2}\right)$ (ICE): 201,50

Precipitación promedio anual (mm): 2 632,52

\section{Subcuenca de la Estación Balsa}

Área de drenaje $\left(\mathrm{km}^{2}\right)$ (Modelo de elevación digital ): 1 620,08

Área de drenaje $\left(\mathrm{km}^{2}\right)$ (ICE): 1638,00

Precipitación promedio anual (mm): 2 206,17

\section{Subcuenca de la Estación Garita}

Área de drenaje $\left(\mathrm{km}^{2}\right)$ (Modelo de elevación digital ): 661,86

Área de drenaje $\left(\mathrm{km}^{2}\right)$ (ICE): 637,20
Precipitación promedio anual (mm): 2 400,31

\subsection{Obtención de caudales para los periodos de retorno definidos}

Las funciones se calcularon en una hoja electrónica.

\subsubsection{Regresión lineal múltiple}

\subsubsection{Ecuación de regresión lineal múltiple}

De acuerdo con los resultados anteriores se establece la ecuación (9) para el caudal Q en $(\mathrm{m} 3 / \mathrm{s})$, para una probabilidad de cien años, con un coeficiente de correlación de 0,963 5 .

\begin{tabular}{lc}
\multicolumn{2}{c}{ Estadísticas de la regresión } \\
Coeficiente de correlación múltiple R & 0,9635 \\
Coeficiente de determinación $\mathrm{R}^{2}$ & 0,9282 \\
& \\
& Coeficiente \\
Intercepción & $-526,9056$ \\
Variable X 1 & 2,2648 \\
Variable X 2 & 0,2082
\end{tabular}

Cuadro 15. Valores de logaritmos de caudal, área y precipitación para un período de retorno de cien años.

\begin{tabular}{|c|c|c|c|c|c|c|c|}
\hline Estación & Número & $\begin{array}{c}\text { Caudal (Q) } \\
\left(\mathrm{m}^{3} / \mathrm{s}\right)\end{array}$ & $\begin{array}{c}\text { Área (A) } \\
\left(\mathbf{k m}^{2}\right)\end{array}$ & $\begin{array}{c}\text { Precipitación media } \\
(\mathbf{P}) \\
(\mathbf{m m} / \mathbf{a} \tilde{n} o)\end{array}$ & $\log Q$ & $\log A$ & $\log P$ \\
\hline Guardia & 741901 & 2487 & 955 & 1747,38 & 3,39568 & 2,98000 & 3,24239 \\
\hline Coyolar & 741906 & 1142 & 128,2 & 1795,08 & 3,05767 & 2,10789 & 3,25408 \\
\hline Palmar & 983101 & 11305 & 4766,7 & 3028,10 & 4,05327 & 3,67822 & 3,48117 \\
\hline Caracaucho & 983107 & 3323 & 1133,3 & 2885,17 & 3,52153 & 3,05434 & 3,46017 \\
\hline Brujo & 983103 & 5629 & 2401,2 & 3118,07 & 3,75043 & 3,38043 & 3,49389 \\
\hline La Cuesta & 983109 & 2509 & 835,5 & 3156,98 & 3,39950 & 2,92195 & 3,49927 \\
\hline Rivas & 983108 & 579 & 317,9 & 3450,78 & 2,76268 & 2,50229 & 3,53792 \\
\hline Amo & 842424 & 1832 & 734 & 2082,74 & 3,26293 & 2,86570 & 3,31864 \\
\hline Tacares & 842402 & 253 & 201,5 & 2632,52 & 2,40312 & 2,30428 & 3,42037 \\
\hline Balsa & 842406 & 1539 & 1638 & 2206,17 & 3,18724 & 3,21431 & 3,34364 \\
\hline Garita & 842412 & 678 & 637,2 & 2400,32 & 2,83123 & 2,80428 & 3,38027 \\
\hline
\end{tabular}


Cuadro 16. Ecuaciones de regresión para el cálculo de caudal máximo anual para diferentes períodos de retorno.

\begin{tabular}{|c|c|c|c|c|c|}
\hline $\begin{array}{l}\text { Período retorno } \\
\text { (años) }\end{array}$ & $\mathbf{r}^{2}$ & $\mathbf{F}$ & $\begin{array}{l}\text { Expor } \\
\mathbf{r}^{2}\end{array}$ & $\begin{array}{l}\text { cial } \\
\mathbf{F}\end{array}$ & $\begin{array}{l}\text { Ecuaciones de caudal máximo anual Q } \\
\qquad\left(\mathrm{m}^{3} / \mathrm{s}\right)\end{array}$ \\
\hline 100 & 0,9282 & 51,75 & 0,7054 & 9,58 & $-526,9056+2,2648 \cdot A+0,2082 \cdot P$ \\
\hline 50 & 0,9342 & 56,76 & 0,7502 & 12,01 & $-466,6341+1,8686 \cdot A+0,2032 \cdot P$ \\
\hline 20 & 0,9399 & 62,58 & 0,8061 & 16,63 & $-398,9397+1,4208 \cdot \mathrm{A}+0,1914 \cdot \mathrm{P}$ \\
\hline 10 & 0,9427 & 65,83 & 0,8457 & 21,92 & $-359,9345+1,1301 \cdot \mathrm{A}+0,1805 \cdot \mathrm{P}$ \\
\hline 2 & 0,9622 & 101,70 & 0,9204 & 46,26 & $-228,1945+0,5417 \cdot \mathrm{A}+0,1140 \cdot \mathrm{P}$ \\
\hline 1 & 0,8990 & 35,62 & 0,9098 & 40,33 & $-194,7543+0,2277 \cdot \mathrm{A}+0,0808 \cdot \mathrm{P}$ \\
\hline
\end{tabular}

$\mathrm{Q}=-526,9056+2,26648 \cdot \mathrm{P}$

\section{Estadísticas de la regresión}

Coeficiente de correlación múltiple $\mathrm{R}$ Coeficiente de determinación $\mathrm{R}^{2}$

Intercepción

Variable X 1

Variable X 2

\section{Regresión Exponencial Múltiple (Cuadro} $15)$.

La ecuación de regresión lineal múltiple tiene un coeficiente de correlación múltiple $\mathrm{R}$ mayor que el de la ecuación exponencial múltiple, y por eso se recomienda el uso de la primera. En forma semejante se obtuvieron las ecuaciones para los demás períodos de retorno (Cuadro 16).

\section{CONCLUSIONES}

1. El análisis estadístico realizado mediante la distribución Log-Person III, a las series de datos de caudal máximo anual de las estaciones en estudio, mostró buenos resultados. Al realizar el estudio de los datos altos y bajos salidos extremos, se encontró que las estaciones Nuestro Amo, y Tacares presentaron valores altos y las estaciones Guardia y Garita presentaron valores bajos, a los cuales se les ejecutó el respectivo ajuste. La integración de Sistemas de Información Geográfica al estudio de cuencas hidrográficas constituye una herramienta de facilitación para la obtención de resultados físicos y el entendimiento de las variables que afectan las condiciones hidrológicas de una cuenca.

2. De acuerdo con los resultados obtenidos en el análisis de regresión, se establece que existe una relación real entre las variables. Debido a la confiabilidad de las muestras de datos y de los resultados obtenidos, las ecuaciones generadas pueden ser utilizadas como un método empírico para el cálculo de caudal para los diferentes períodos de retorno analizados.

3. Al realizar el análisis de regresión lineal y el de exponencial múltiple para cada una de las probabilidades en estudio, en el análisis lineal se obtuvieron valores para el coeficiente de correlación más cercanos a uno que en el análisis exponencial. De esta manera para las probabilidades de cien, cincuenta, veinte, diez y dos años, la ecuación de caudal tendrá una relación lineal, para la probabilidad de un año no se presentó esta situación, pero en vista de que no hay mucha diferencia entre los valores del coeficiente de correlación se consideró, que por homogeneidad en los resultados, la ecuación será también de índole lineal.

4. Aunque las tres cuencas analizadas pertenecen a la vertiente Pacífica, todas pertenecen a diferentes zonas climáticas y tienen formaciones geológicas y topografías muy variadas, estos factores influyen directamente en el cálculo del 
caudal; mas aún, al realizar el análisis de regresión para los resultados de caudal, área y precipitación para las once estaciones en estudio se concluye que hay muy buena relación entre la variable dependiente (correspondiente al caudal) y las variables independientes (área y precipitación).

\section{AGRADECIMIENTOS}

Para la realización del trabajo fue imprescindible la colaboración del Instituto Costarricense de Electricidad, por medio del Lic. SadíL Laporte, el cual brindó la información hidrológica de las estaciones que se analizaron, así como la información meteorológica de las estaciones que se ubican dentro de las mismas. Por otro lado, también se contó con la colaboración del Instituto Meteorológico Nacional, por medio del Ing. Roberto Villalobos, donde se suministraron los datos de precipitación de las estaciones complementarias a las que tenía el ICE y los Modelos de elevación digital de las cuencas en estudio.

\section{REFERENCIAS BIBLIOGRÁFICAS}

Interagency Advisory Committee on Water Data. (1982). Guidelines for determining flood flow frequency. Bulletin 17B, US Dept. generated by mixed processes. Water Resources Research

Instituto Costarricense de Electricidad (ICE). (2004). Información limnigráfica y meteorológica de las cuencas de la vertiente del pacífico: río Tempisque, río Grande de Tárcoles y río Grande de Térraba. Registros de 1951 al 2001.

Instituto Meteorológico de Costa Rica (IMN). (2004). Información meteorológica de las cuencas de la vertiente del Pacífico: río Tempisque, río Grande de Tárcoles y río Grande de Térraba. Registros de 1951 al 2001.

\section{SOBRE LA AUTORA Y LOS AUTORES}

\section{Nazareth Rojas Morales}

Licenciada en Ingeniería Agrícola de la Universidad de Costa Rica

Instituto Meteorológico de Costa Rica.

Correo electrónico: nrojas@imn.ac.cr

\section{José Francisco Aguilar Pereira}

Licenciado en Ingeniería Agrícola de la Universidad de Costa Rica

Magister Scientiae en Computación, Sistema de información Instituto tecnológico de Costa Rica Correo electrónico: jaguilar@ingagri.ucr.ac.cr

\section{Hernán Solís Bolaños}

Licenciado en Ingeniería Civil de la Universidad de Costa Rica M. Sc. del Silsoe College de Inglaterra. Doctor en Hidráulica del Instituto de Construcciones de Bucarest, Rumania. Correo electrónico: hsolis48@yahoo.com 